\title{
TRIM16 acts as a tumour suppressor by inhibitory effects on cytoplasmic vimentin and nuclear E2F1 in neuroblastoma cells
}

\author{
GM Marshall ${ }^{1,2}$, JL Bell ${ }^{1}$, J Koach ${ }^{1}$, O Tan ${ }^{1}$, P Kim ${ }^{1}$, A Malyukova ${ }^{1}$, W Thomas ${ }^{1}$, EO Sekyere ${ }^{1}$,

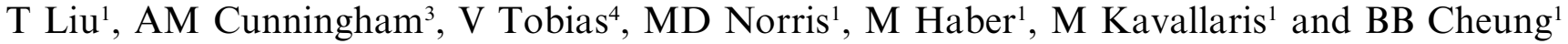 \\ ${ }^{1}$ Children's Cancer Institute Australia for Medical Research, Randwick, New South Wales, Australia; ${ }^{2}$ Centre for Children's Cancer \\ and Blood Disorders, Sydney Children's Hospital, Randwick, New South Wales, Australia; ${ }^{3}$ School of Women's and Children's \\ Health, Faculty of Medicine, UNSW, New South Wales, Australia and ${ }^{4}$ Paediatric Anatomical Pathology, South Eastern Area \\ Laboratory Services, Sydney Children's Hospital, Randwick, New South Wales, Australia
}

The family of tripartite-motif (TRIM) proteins are involved in diverse cellular processes, but are often characterized by critical protein-protein interactions necessary for their function. TRIM16 is induced in different cancer types, when the cancer cell is forced to proceed down a differentiation pathway. We have identified TRIM16 as a DNA-binding protein with histone acetylase activity, which is required for the retinoic acid receptor $\beta_{2}$ transcriptional response in retinoid-treated cancer cells. In this study, we show that overexpressed TRIM16 reduced neuroblastoma cell growth, enhanced retinoid-induced differentiation and reduced tumourigenicity in vivo. TRIM16 was only expressed in the differentiated ganglion cell component of primary human neuroblastoma tumour tissues. TRIM16 bound directly to cytoplasmic vimentin and nuclear E2F1 in neuroblastoma cells. TRIM16 reduced cell motility and this required downregulation of vimentin. Retinoid treatment and enforced overexpression caused TRIM16 to translocate to the nucleus, and bind to and downregulate nuclear E2F1, required for cell replication. This study, for the first time, demonstrates that TRIM16 acts as a tumour suppressor, affecting neuritic differentiation, cell migration and replication through interactions with cytoplasmic vimentin and nuclear E2F1 in neuroblastoma cells.

Oncogene (2010) 29, 6172-6183; doi:10.1038/onc.2010.340; published online 23 August 2010

Keywords: TRIM16; EBBP; neuroblastoma; vimentin; E2F1; tumour suppressor

\section{Introduction}

The tripartite motif (TRIM) protein family is composed of more than 70 highly conserved proteins, which display three similar structural motifs: N-terminal, RING-B box-coiled-coil domains and a variable

Correspondence: Dr BB Cheung, Molecular Carcinogenesis Program, Children's Cancer Institute Australia, Lowy Cancer Research Centre, University of NSW, High Street (PO Box 81), Randwick, New South Wales 2031, Australia.

E-mail: bcheung@ccia.unsw.edu.au

Received 16 December 2009; revised 14 May 2010; accepted 30 June 2010; published online 23 August 2010
C-terminus. TRIM proteins have been ascribed important roles in development, ubiquitination, retroviral immunity and cancer, but commonly depend on protein-protein interactions to fulfil their cellular functions (Le Douarin et al., 1995; Cao et al., 1998; Meroni et al., 2005; Vichi et al., 2005).

TRIM16, also called the oestrogen-responsive B box protein, is a member of the RING-B box-coiled-coil/ TRIM protein family, and is constitutively expressed in many tissues in a nascent and post-translationally modified form (Liu et al., 1998). In contrast to most B-box family members, TRIM16 has a predominantly cytoplasmic localization. TRIM16 is missing the RING finger domain, but has, in addition, a C-terminal Ret finger, or B30.2 (SPRY), domain of unknown function. This is shared with several other tripartite motif proteins, such as Midline 1 (MID1/TRIM 18) and TRIM 20, mutated in the human disorders Opitz syndrome and Familial Mediterranean fever, respectively (Trockenbacher et al., 2001; Chae et al., 2003).

In search for factors that mediate the retinoid anticancer signal, we have shown that the TRIM16 protein binds DNA and has histone acetyltransferase activity, which is required for the retinoid-induced retinoic acid (RA) receptor $\beta_{2}$ transcriptional response and growth inhibition in retinoid-treated cancer cells (Cheung et al., 2006; Raif et al., 2009). TRIM16 is a ubiquitously expressed protein that is predominantly cytoplasmic, and undergoes serine-threonine phosphorylation and increased protein stability following retinoid treatment. TRIM16 expression is also induced by oestrogen in breast cancer cells and keratinocyte growth factor in keratinocytes, as cells are entrained to undergo differentiation (Liu et al., 1998; Beer et al., 2002). TRIM16 reduced the cell viability in retinoid-sensitive neuroblastoma cells and retinoid-resistant breast and lung cancer cells, whereas the viability of non-cancer cells was unaffected by TRIM16 overexpression (Cheung et al., 2006; Raif et al., 2009). Importantly, exogenous TRIM16 overexpression in retinoid-resistant cancer cells induced retinoid sensitivity, making TRIM16 an important target for future differentiation therapy development in cancer.

Neuroblastoma is the most common solid tumour of early childhood, originating from cells that form the 
normal sympathetic nervous system. The tumour cells have embryonic features, presumably as a consequence of an impaired capacity to respond to signals operating during normal differentiation. RA causes ganglionic differentiation of neuroblastoma cell lines in vitro, characterized by the appearance of long neurites and cell growth arrest (Thiele et al., 1985). This observation led to successful clinical trials in children with advanced neuroblastoma, showing that retinoid therapy significantly improved survival in these patients (Matthay et al., 1999, 2009).

The signalling pathways and mechanisms underpinning the effect of TRIM16 in retinoid-treated cancer cells are yet to be fully delineated. Owing to the profound effects of TRIM16 overexpression in the presence of retinoids, we examined the hypothesis that TRIM16 may act as a tumour suppressor in the absence of retinoids, and, moreover, that protein-protein interactions might explain its cellular effects. In this study, we show that TRIM16 is an important regulator of neuroblastoma cell differentiation, migration and tumourigenicity, via its interaction with cytoplasmic vimentin and nuclear E2F1 proteins in neuroblastoma cells.

\section{Results}

TRIM16 reduces neuroblastoma cell viability, clonogenicity and tumourigenicity

We have previously shown that TRIM16 can enhance the retinoid anti-proliferative effect on cancer cells through transcriptional regulation of retinoic acid receptor $\beta_{2}$. In this study, we sought to investigate whether TRIM16 had a tumour suppressor function in neuroblastoma, independent of its effects on the retinoid anticancer signal. We derived stable neuroblastoma BE(2)-C cell lines, which overexpressed TRIM16 (Figure 1a). In an Alamar Blue assay (Figure 1b), overexpression of TRIM16 in neuroblastoma tumour cells decreased the cell viability in two independently derived clones (TRIM16-4 and TRIM16-5), compared with empty vector clones $(\mathrm{EV}-1$ and $\mathrm{EV}-8)(P<0.001)$. Anchorage-independent growth in soft agar was also reduced in TRIM16-expressing clones, compared with control clones $(P<0.01)$ (Figures 1c and $\mathrm{d})$. In a xenograft BALB/c nude mouse model, TRIM16 overexpression led to substantially decreased neuroblastoma tumour growth, as compared with the empty vector controls, 4 weeks post engraftment $(P<0.01)$ (Figure 1e). Together, these data indicated that TRIM16 inhibited the tumourigenic phenotype of neuroblastoma tumour cells in the absence of excess retinoid. Furthermore, we examined the effect of knockdown of TRIM16 expression on cell growth. The efficiency of TRIM16 small interfering RNA (siRNA) was evaluated after 24, 48 and $72 \mathrm{~h}$ of transfection by western blot $(70 \%$ knockdown). The cell viability of $\mathrm{BE}(2)-\mathrm{C}$ was significantly increased after transfection of $20 \mathrm{~nm}$ siRNA TRIM16 when compared with control siRNA $(P<0.001)$ (Figure 1f).
TRIM16 induces neuronal differentiation

in neuroblastoma cells

TRIM16 expression is increased by oestrogen, keratinocyte growth factor and retinoic acid in breast cancer, skin and neuroblastoma cells, respectively (Liu et al., 1998; Beer et al., 2002; Cheung et al., 2006). In order to investigate the mechanism of the TRIM16 tumour suppressor function, TRIM16-expressing BE(2)-C neuroblastoma cell clones were examined for neuritic differentiation in vitro in the presence of retinoid, and compared with control clones. Overexpression of TRIM16 led to a significant increase in the proportion of neurite-bearing cells after $10 \mu \mathrm{M}$ all-trans-retinoic acid (aRA) treatment at days 4 and 6, compared with empty vector controls $(P<0.001)$ (Figures $2 \mathrm{a}$ and $\mathrm{b}$ ).

Neuroblastoma tumour tissue is composed of primitive neuroblasts, more differentiated mature ganglion cells and interstitial neuropil. Moreover, a high degree of ganglion cell maturation within a tumour correlates with a better patient prognosis (Joshi, 2000). We therefore wanted to investigate whether TRIM16 was selectively expressed in particular cell types within the neuroblastoma tumour tissue. Paraffin-embedded, formalin-fixed human neuroblastoma tumour cross-sections were examined for the pattern of endogenous TRIM16 expression by immunohistochemical staining using a TRIM16-specific antibody in 21 patients from five clinical stages (stage I, II, III, IV and IVS). The expression of TRIM16 was stronger in the large ganglion cells and interstitial neuropil, which comprise the more differentiated component of the tumour, and is largely absent in the smaller primitive neuroblasts (Figure 2c). The proportion of TRIM16-positive cells was significantly higher in the differentiated region, compared with the poorly differentiated region $(P<0.001)$ across all clinical stages (Figure $2 \mathrm{~d})$. However, there was no statistically significant relationship between clinical stage and TRIM16 protein expression. We confirmed the differentiated nature of the region of the neuroblastoma tumour shown in Figure 2e, by demonstrating co-expression of the neuronal marker, class III $\beta$-tubulin isotype (Katsetos et al., 1991). Furthermore, immunohistochemical staining revealed a strong nuclear and cytoplasmic TRIM16 expression in neonatal differentiated superior cervical ganglia cells, coeliac ganglia cells and normal adrenal gland. In contrast, TRIM16 expression is reduced in poorly differentiated neuroblastoma tumour cells and is predominately in the cytoplasm of 6-week-old TH-MYCN transgenic mouse tumours (Weiss et al., 1997).

\section{TRIM16 binds to and modulates vimentin protein} expression in both neuroblastoma and lung cancer cells TRIM proteins commonly bind to other proteins to affect cellular function (Urano et al., 2002); thus, we investigated TRIM16-binding proteins in neuroblastoma cells. We examined protein lysates from BE(2)-C cells, transiently transfected with full-length TRIM16 tagged with an MYC oligopeptide for $48 \mathrm{~h}$. As a negative control, we compared lysates from cells that 


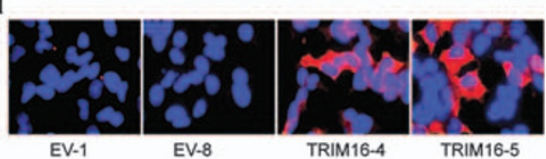

b

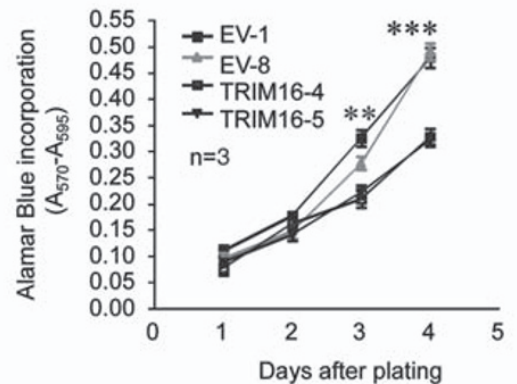

d

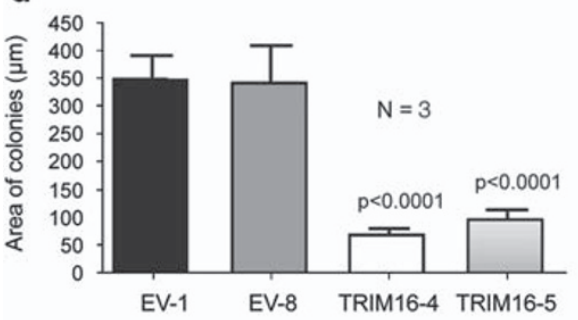

f

siRNA Control TRIM16 Control TRIM16 Control TRIM16

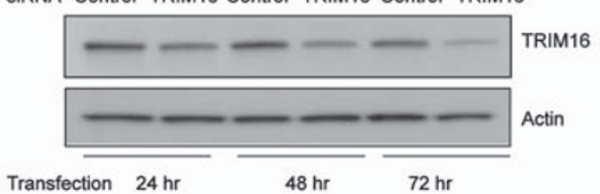

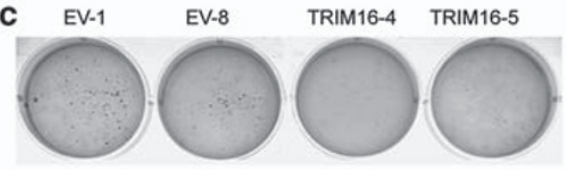
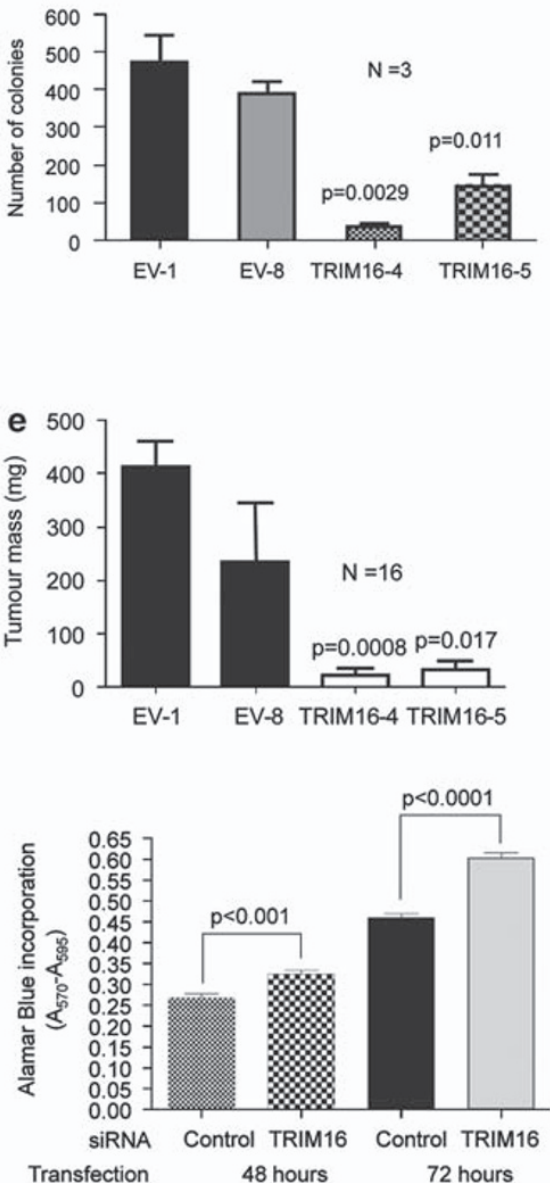

Figure 1 TRIM16 reduces neuroblastoma cell viability, clonogenicity and tumourigenicity. (a) The cells were stained with an antibody recognizing the Myc-tag epitope to confirm the TRIM16 plasmid transfection by immunofluorescence microscopy and immunoblot examination. (b) The viable cells were measured up to day 4 by Alamar Blue assay in BE(2)-C cells stably transfected with empty vector or with TRIM16 plasmid DNA. A statistically significant difference $(* * P<0.01$ and $* * * P<0.001)$ compared with the empty vector control. (c) In the top panel, the clonogenicity of various BE(2)-C cell transfectants was determined using soft agar. In the bottom panel, number of colonies were compared between empty vector control (EV-1) and TRIM16 plasmid transfected BE(2)-C cell. (d) A colony-forming assay showed reduced colony size in clones overexpressing TRIM16. A statistically significant difference $(P<0.0001)$ compared with empty vector control (EV-1). (e) The tumour volume of TRIM16 or empty vector transfected BE(2)-C cells injected into nude mice for 4 weeks. Each value shown in the figure represented data from 16 mice. A statistically significant difference $(P<0.01)$ compared with empty vector control $(\mathrm{EV}-1)$. (f) In the left panel, western blotting analysis by TRIM16-specific antibody at 24, 48 and $72 \mathrm{~h}$ of $\mathrm{BE}(2)-\mathrm{C}$ cells without or with TRIM16 siRNA transfection. Actin was used as an internal control for loading. In the right panel: the viable cells were measured up to $72 \mathrm{~h}$ by Alamar Blue assay in BE(2)-C cells transfected with control siRNA or TRIM16 specific siRNA.

were transfected with an empty vector. The supernatant was immunoprecipitated by an anti-MYC-Tag antibody. Protein bands were cut directly from the gel and micro-sequenced by the liquid chromatography-tandem mass spectrometry (LC-MS/MS) analyses. We have identified seven candidate TRIM16-binding partners (vimentin, E2F1, prohibitin, TRAP-1, EBNA2 coactivator p100, EPLIN and scaffold attachment factor A) in this way. We then performed immunoprecipitation and immunoblotting experiments on four of the candidate binding partners, and confirmed that vimentin, E2F1 and prohibitin indeed interact with TRIM16 in $\mathrm{BE}(2)-\mathrm{C}$ cells. As vimentin expression is not only a marker associated with the invasive phenotype of cancer, but is also required for carcinoma cell motility, and E2F1 is a transcription factor that is central for cell survival and proliferation, vimentin (a cytoplasmic protein) and E2F1 (a nuclear protein) were chosen for further studies.

Vimentin is the predominant intermediate filament protein in mesenchymal cells, and is implicated in metastasis and cancer cell migration in colon and breast cancer cell lines (McInroy et al., 2007). To determine whether TRIM16 interacted with vimentin, co-immunoprecipitation (co-IP) of vimentin with Myc-TRIM16 


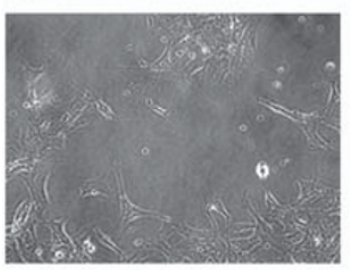

$\mathrm{EV}-1$

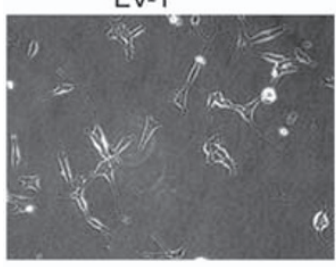

TRIM16-4

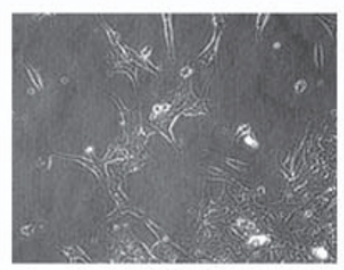

EV-8

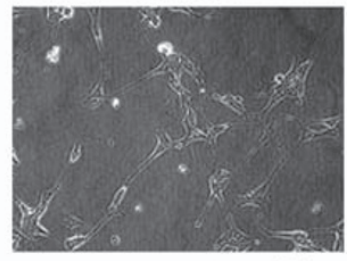

TRIM16-5 $200 \mu \mathrm{m}$
C

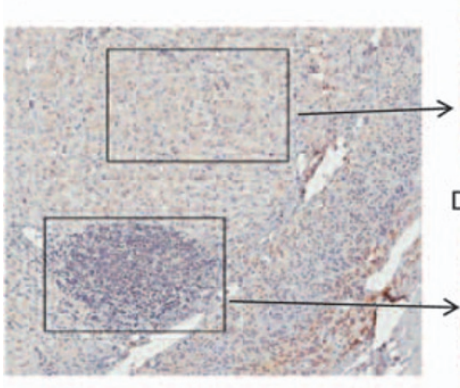

Neuroblastoma tumour tissue, stage I

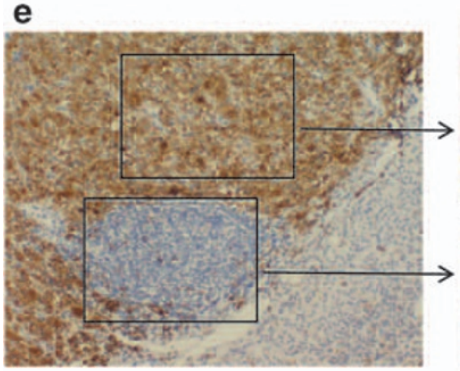

Neuroblastoma tumour tissue
Poorly differentiated

region

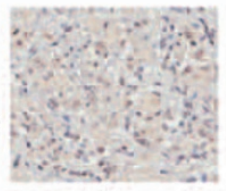

Differentiated region
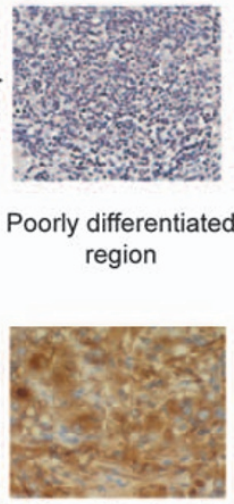

Differentiated region

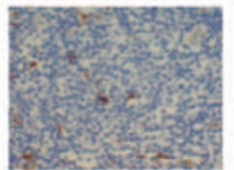

Poorly differentiated

region b

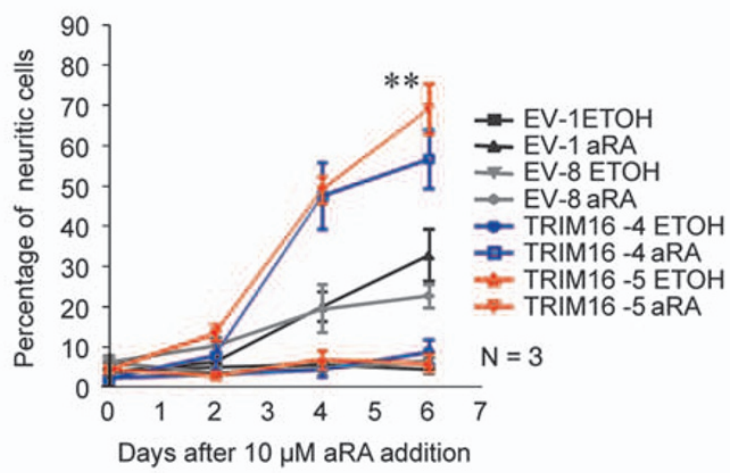

d

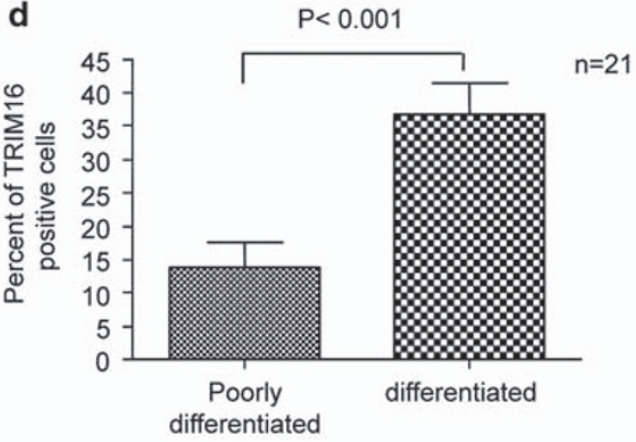

f Tumour

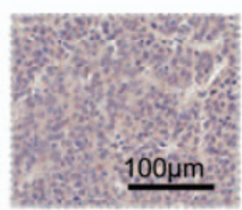

Para-vertebra Ganglia

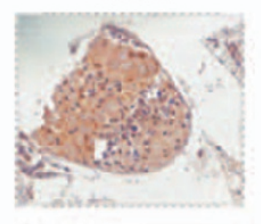

Adrenal

Ganglia

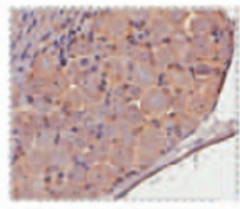




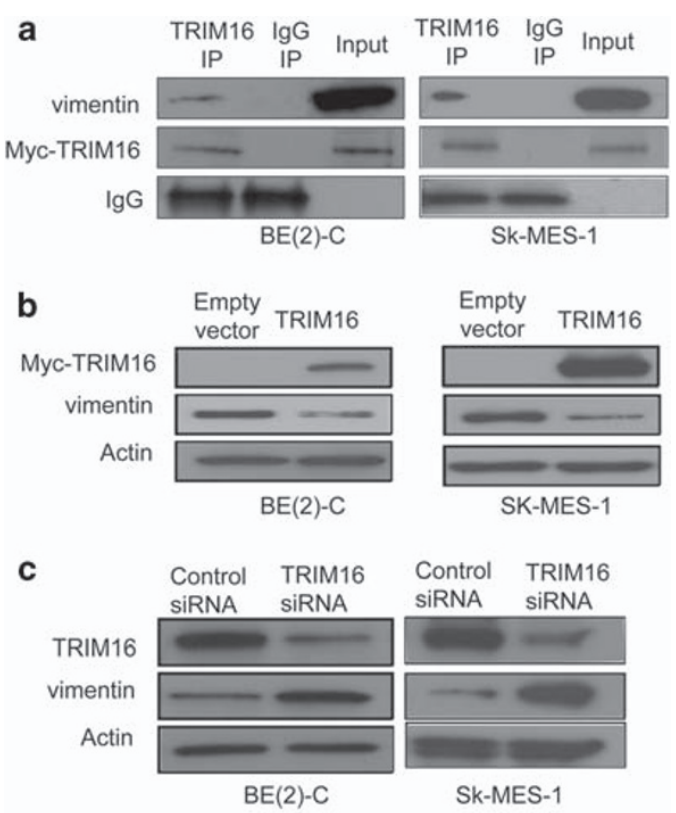

d
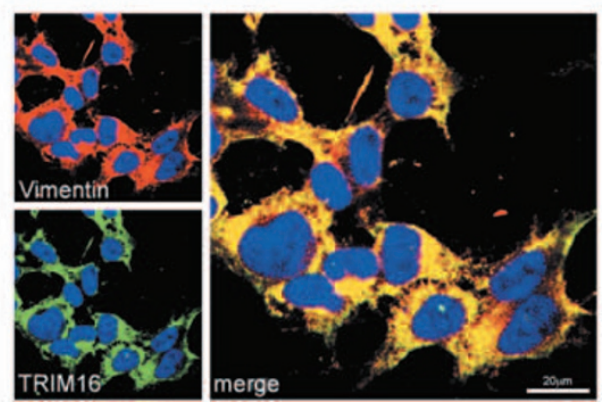

Figure 3 TRIM16 binds to and modulates vimentin protein expression in both neuroblastoma and lung cancer cells. (a) Interaction of TRIM16 with vimentin. Lysates of $\mathrm{BE}(2)-\mathrm{C}$ and SK-MES-1 cells transfected with TRIM16 plasmids were analysed by immunoblotting using anti-vimentin and anti-Myc-tag antibodies (western blots: vimentin and TRIM16 proteins), combined without (input) or with immunoprecipitation using anti-TRIM16 antibody or anti-IgG antibody control. (b) TRIM16 overexpression downregulates exogenous vimentin. Lysates of transfected BE(2)-C and SK-MES-1 cells were analysed by antiTRIM16 (top panel) or anti-vimentin (middle panel) or anti-actin as loading control (bottom panel). (c) BE(2)-C and SK-MES-1 cells were transfected with either control siRNA or siRNA specifically targeting TRIM16 for $72 \mathrm{~h}$, followed by protein extraction and western blot. (d) Immunocytochemical expression patterns vimentin (orange/red fluorescence) and TRIM16 (green fluorescence) in $\mathrm{BE}(2)-\mathrm{C}$ cells colocalized in the cytoplasm (visualized as yellow fluorescence in merge panel).

extent in the nucleus of COS- 1 and HaCaT cells. The localization of endogenous TRIM16 and vimentin proteins in neuroblastoma cells was determined by confocal microscopy. In Figure 3d, the merged image of vimentin and TRIM16 expression showed that the endogenous proteins were colocalized to the cytoplasm.

Downregulated vimentin is required for TRIM16 effects on cell motility and migration

We next tested the hypothesis that TRIM16 overexpression may reduce cell motility via its interaction a

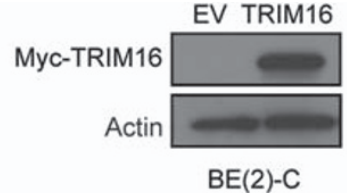

Empty vector
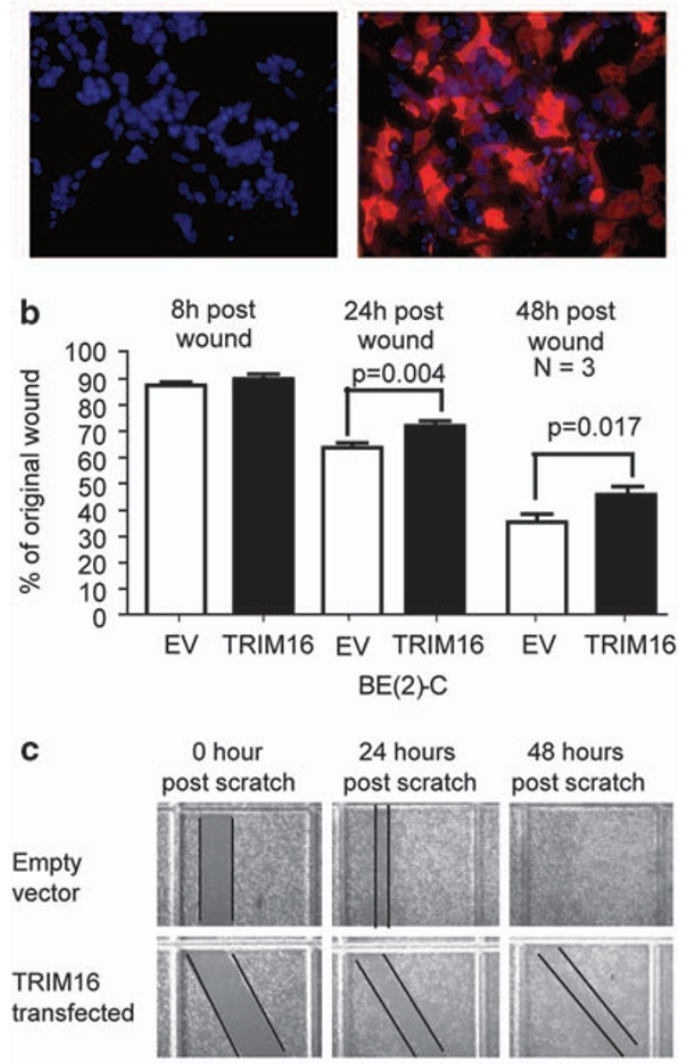

48 hours post scratch

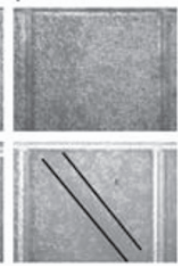

$B E(2)-C$

Figure 4 Downregulated vimentin is required for TRIM16 effects on cell motility. (a) Lysates of $\mathrm{BE}(2)-\mathrm{C}$ cells transfected with TRIM16 plasmids were analysed by immunoblotting and an immunofluorescence assay using anti-Myc antibody. (b) Relative closure of $\mathrm{BE}(2)-\mathrm{C}$ wounds. The average distance moved by the empty vector control and the average (and s.e.) movement of TRIM16 plasmid DNA transfected cells in three independent wounds is shown relative to the percentage of original wound. Three locations of three independent empty vector control and TRIM16 plasmid DNA transfected wounds were photographed at 8,24 and $48 \mathrm{~h}$ post wound. (c) Representative phase contrast micrographs of closure of scratch-wounded confluent cultures of empty vector or TRIM16 plasmid DNA transfected BE(2)-C cells at time point immediately after wounding and 24 and $48 \mathrm{~h}$ post wounding.

with vimentin in neuroblastoma cells. We transiently transfected TRIM16 plasmid DNA and empty vector control into BE(2)-C neuroblastoma cells. To optimize transfection conditions, we measured the effects of increasing amounts of plasmid DNA, while maintaining a constant volume of lipofectamine. Maximum efficiency was found to occur between 10 to $15 \mu \mathrm{g}$ of plasmid per $1 \times 10^{6}$ cells in T75 flasks; $70 \%$ of cells were typically TRIM16-myc positive (Figure 4a). The scratch- 
a

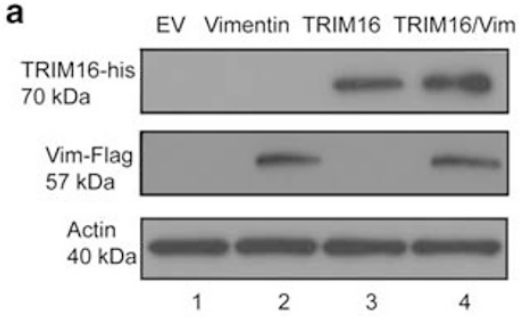

C

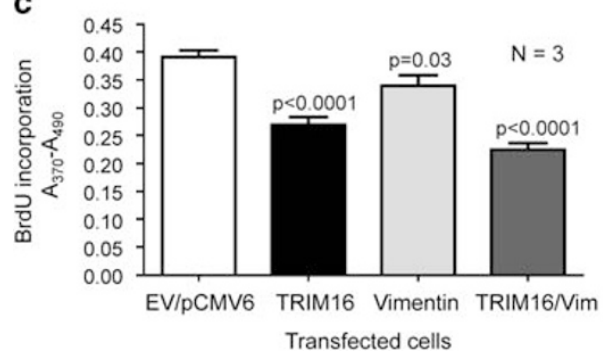

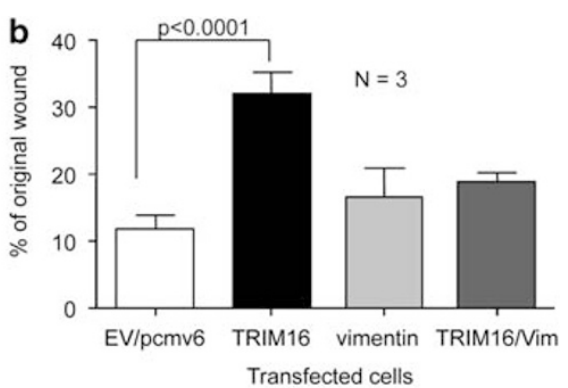

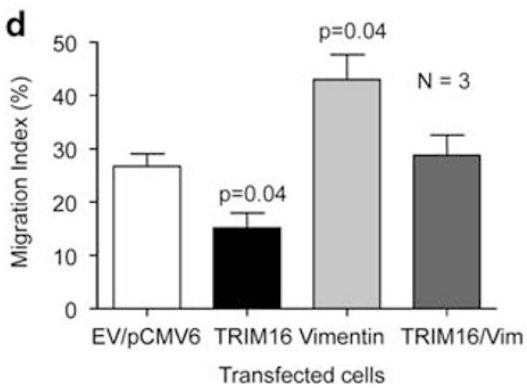

Figure 5 Downregulated vimentin is required for TRIM 16 reduction of cell motility and cell migration. (a) Lysates of BE(2)-C cells transfected with indicated plasmids (lane 1: pcDNA3.1 and pCMV-6 empty vectors; lane 2: vimentin; lane 3: TRIM16; and lane 4: TRIM16/vimentin) were analysed by immunoblotting using anti-His and anti-Flag antibodies. (b) The average distance migrated by the two empty vector controls (lane 1), TRIM16 (lane 2), vimentin (lane 3) and TRIM16/vimentin (lane 4) plasmid DNA-transfected cells in three independent wounds is shown relative to the percentage of original wound. (c) BE(2) cells were transfected with different plasmid DNA for $72 \mathrm{~h}$, followed by incubation with 5-bromo-2-deoxyuridine (BrdU) for the last $6 \mathrm{~h}$. BrdU incorporation was measured as OD units of absorbance. (d) Invasion assay of BE(2)-C cells through collagen-coated cell culture inserts. The percentage of the migrated cells divided by the total number of cells in the wells from three independent experiments is shown.

wound assays on confluent monolayers were used to investigate cell motility (McInroy et al., 2007). The BE(2)C cells, which overexpressed TRIM16, remained largely open at multiple time points, while empty vector control cells migrated particularly rapidly in this assay, filling the empty wound space (Figures $4 \mathrm{~b}$ and $\mathrm{c}$ ).

To investigate whether repression of vimentin expression by TRIM16 is required for cell motility and proliferation in neuroblastoma cells, we transiently transfected both TRIM16 and vimentin plasmid DNA into $\mathrm{BE}(2)-\mathrm{C}$ cells for $48 \mathrm{~h}$, and quantified wound closure at $32 \mathrm{~h}$ post-scratch. In Figure $5 \mathrm{a}$, the anti-Histag antibody and anti-Flag-tag antibody were used to confirm exogenous TRIM16 and vimentin overexpression, respectively. Overexpression of TRIM16 caused significantly slower wound closure at $32 \mathrm{~h}$, compared with vimentin transfectants and empty vector control $(P<0.0001)$ (Figure 5b). Significantly, ectopic overexpression of both TRIM16 and vimentin into $\mathrm{BE}(2)-\mathrm{C}$ cells blocked the inhibitory effect of TRIM16 on wound closure (Figure 5b). In contrast, overexpressed vimentin did not abrogate the inhibitory effect of TRIM16 on cell proliferation (Figure 5c). To investigate the role of TRIM16 and vimentin in neuroblastoma cell migration, we examined the invasiveness of $\mathrm{BE}(2)-\mathrm{C}$ cells in a Transwell plate migration assay using $10 \%$ fetal calf serum as a chemoattractant. As shown in Figure 5d, overexpression of TRIM16 demonstrated slower cell migration at $24 \mathrm{~h}$ compared with the empty vector control $(P=0.04)$. In contrast, overexpression of vimentin demonstrated increased cell migration at $24 \mathrm{~h}$ compared with empty vector control $(P=0.04)$.
Significantly, ectopic overexpression of both TRIM16 and vimentin into $\mathrm{BE}(2)-\mathrm{C}$ cells restored the cell migration index to control levels. These data indicate that repression of vimentin expression is specifically required for TRIM16 to exert its inhibitory effects on cell migration.

\section{TRIM16 translocates to the nucleus and decreases nuclear} E2F1 levels in neuroblastoma cells

Our LC-MS/MS analysis identified the nuclear transcription factor and cell cycle regulatory protein, E2F1 (Johnson et al., 2006), as a potential TRIM16-binding protein. We hypothesized that TRIM16 might also translocate to the nucleus and directly interact with E2F1 to affect neuroblastoma cell proliferation. To test this hypothesis, $\mathrm{BE}(2)-\mathrm{C}$ neuroblastoma cells were transiently transfected with TRIM16 plasmid DNA; then, $24 \mathrm{~h}$ later, nuclear and cytoplasmic protein fractions were subjected to co-IP with a Myc-tagged antibody, which recognized the transfected TRIM16. Co-IP confirmed that transfected TRIM16 indeed formed a complex with endogenous E2F1 in neuroblastoma cells (Figure 6a). To determine whether TRIM16 regulates $\mathrm{E} 2 \mathrm{~F} 1$ protein expression in the nucleus, $\mathrm{BE}(2)$ $\mathrm{C}$ cells were transiently transfected with TRIM16 plasmid DNA, or treated with $10 \mu \mathrm{M}$ aRA for $48 \mathrm{~h}$. As shown in Figures $6 \mathrm{~b}$ and $\mathrm{c}$, the amount of TRIM16 protein in the nucleus was increased in cells after both aRA treatment and TRIM16 transfection, when compared with untreated control cells. Following aRA treatment, TRIM16 exhibited marked nuclear 
a

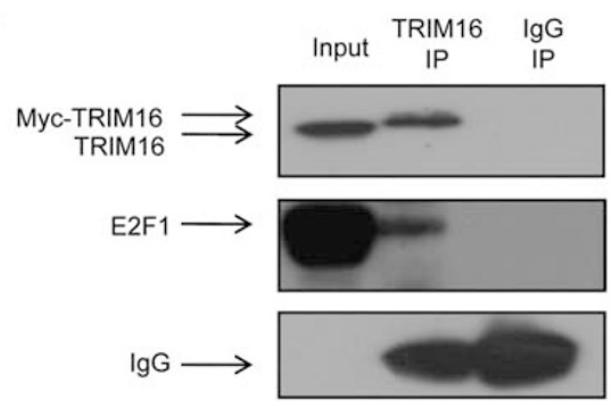

Nuclear protein from BE(2)-C

C
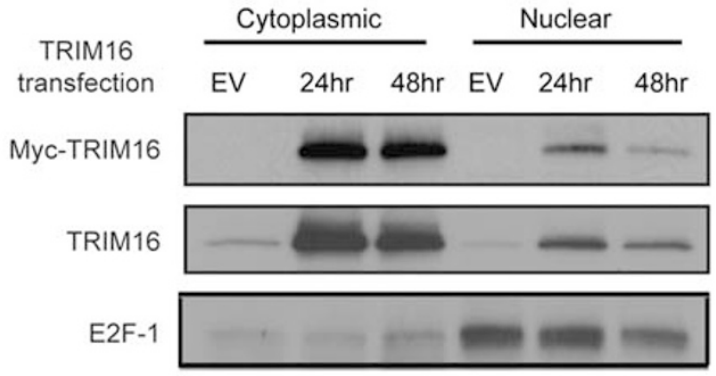

GAPDH

Actin
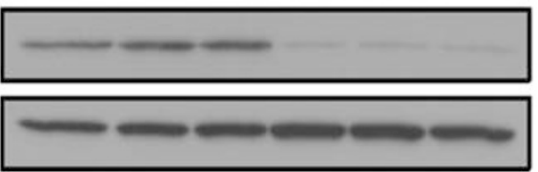

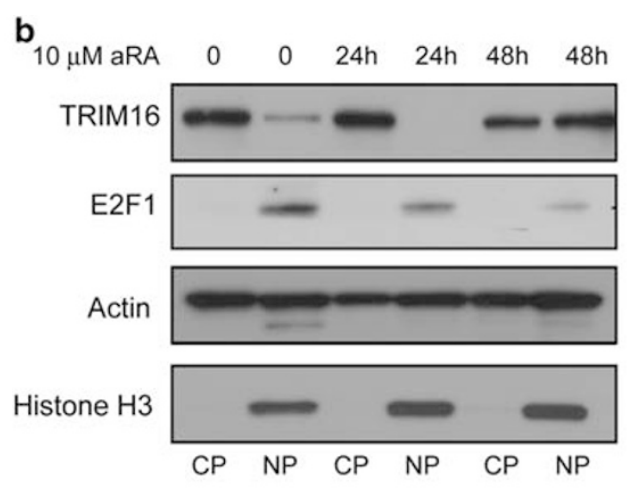

d

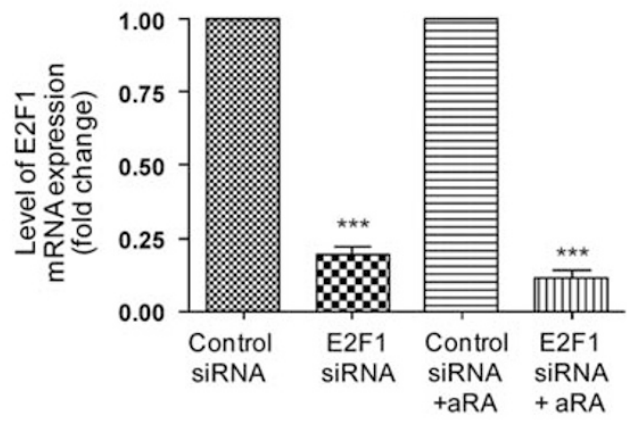

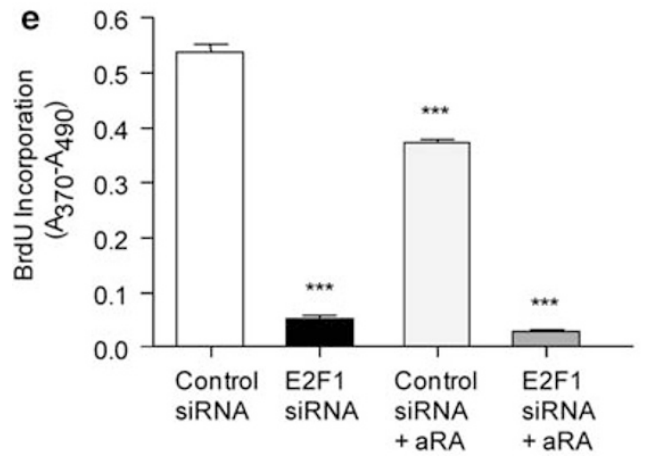

f

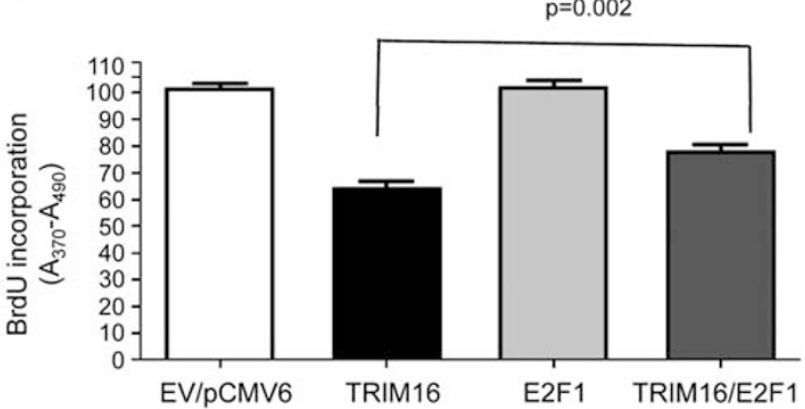

Figure 6 TRIM16 translocates to the nucleus and decreases E2F1 levels in BE(2)-C cells. (a) The nuclear protein extract from BE(2)$\mathrm{C}$ cells transfected with TRIM16 plasmids was incubated with either a mouse IgG (right lane) or an anti-Myc antibody (middle lane). The purified IP complex was analysed by western blot using antibodies for TRIM16 and E2F1 proteins. (b, c) Immunoblotting analysis of expression of TRIM16 and E2F1 in BE(2)-C cells. Cytoplasmic (CP) and nuclear (NP) proteins of cells treated with $10 \mu \mathrm{M}$ all-transretinoic acid (aRA) for 24 and $48 \mathrm{~h}$ (Figure $6 \mathrm{~b}$ ) or transfected with TRIM16 plasmid DNA for 24 and $48 \mathrm{~h}$ (Figure 6c) were analysed by immunoblotting using anti-TRIM16 and anti-E2F1 antibodies. Anti-actin antibody is used as loading control, anti-histone H3 is used as control for nuclear protein and anti-GAPDH antibody is used as cytoplasmic protein control. (d, e) BE(2)-C cells were transfected with control siRNA or siRNA specifically targeting E2F1 for $72 \mathrm{~h}$, followed by real-time PCR and 5-bromo-2-deoxyuridine (BrdU) cell proliferation assay. A statistically significant difference $(* * * P<0.001)$ compared with control siRNA. (f) BE(2) cells were transfected with different plasmid DNA for $72 \mathrm{~h}$, followed by incubation with BrdU for the last $6 \mathrm{~h}$. BrdU incorporation was measured as OD units of absorbance.

translocation at $48 \mathrm{~h}$, coinciding with a reduction in nuclear E2F1 (Figure 6b). In neuroblastoma cells transfected with TRIM16, there was an even greater increase in overall cellular TRIM16, with some of the excess TRIM16 moving to the nucleus. Interestingly, the transfected nuclear TRIM16 levels fell quickly to basal levels by $48 \mathrm{~h}$ post-transfection. Higher nuclear TRIM 16 levels post-transfection again correlated with a twofold reduction in nuclear E2F1 levels at $48 \mathrm{~h}$ (Figure 6c). The level of E2F1 mRNA did not change in BE(2)-C cells with overexpression of TRIM16 (data not shown).

To determine whether the higher basal E2F-1 levels in neuroblastoma cells affected cell proliferation, we transiently transfected siRNAs targeting E2F1 or control siRNA into $\mathrm{BE}(2)-\mathrm{C}$ cells, in the presence or absence of $10 \mu \mathrm{M}$ aRA for $72 \mathrm{~h}$. E2F1 siRNAs knocked down E2F1 expression and reduced cell proliferation by more than $90 \%$ in both aRA-treated and untreated cells 


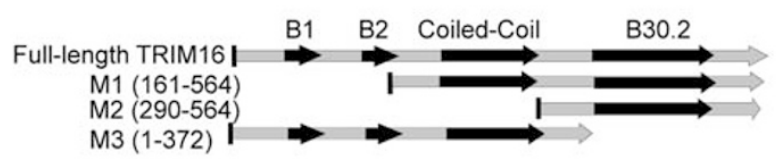

b

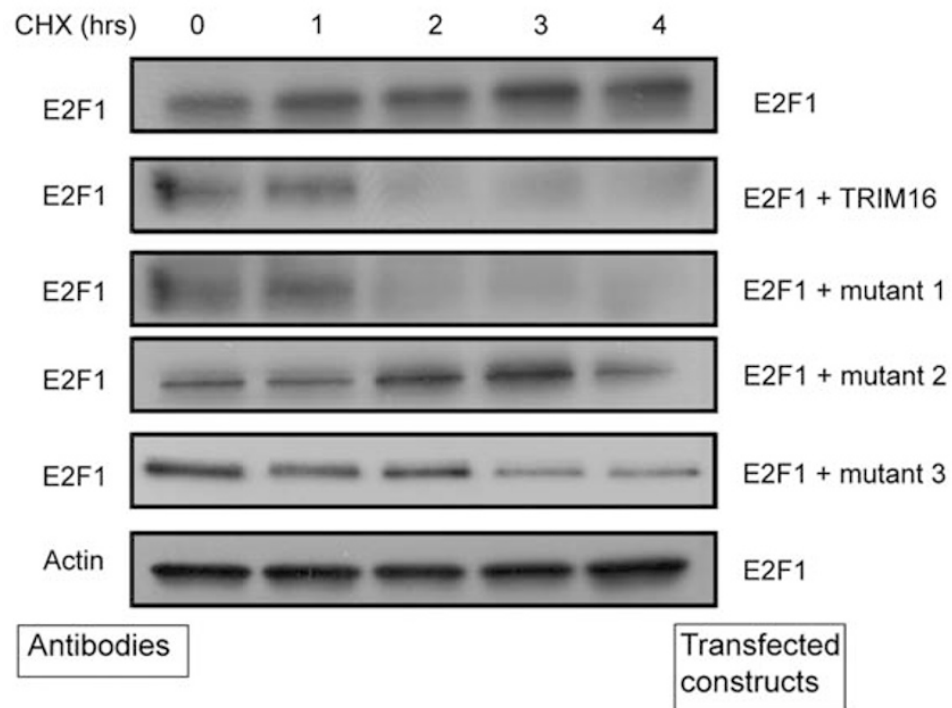

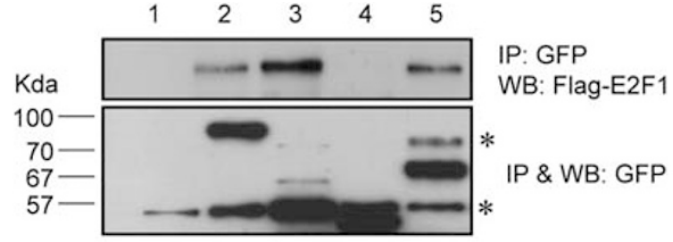

EV Full M1 M2 M3
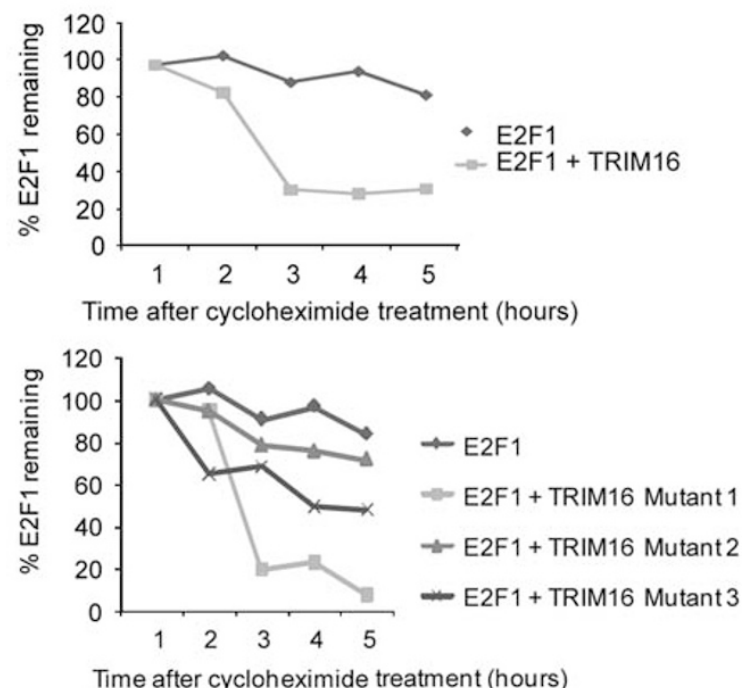

Figure 7 Identification of the interaction domains of TRIM16 and E2F1. (a) Interaction of individual TRIM16 domains with E2F1: Flag-tagged E2F1 and individual domains of TRIM16 were coexpressed in BE(2)-C cells. Flag- and GFP-tagged immunoprecipitates (IPs) were analysed for the presence of Flag- and GFP-tagged constructs (co-IPs) by western blots. Transfection of E2F1 together with the empty vector was used as a control. Non-specific bands are labelled by asterisks. (b) TRIM16 downregulates the protein E2F1 level by reducing the protein's half-life: BE(2)-C cells were transfected either with E2F1 or TRIM16, as well as E2F1 and TRIM16 mutants where indicated. After $16 \mathrm{~h}$ the transfected cells were trypsinized, pooled and then replated. After $24 \mathrm{~h}$, cycloheximide was added at a final concentration of $25 \mu \mathrm{g} / \mathrm{ml}$ to the media. At the specified time points the cells were harvested and the protein extracted for analysis by western blots. The western blots were probed with polyclonal rabbit anti-E2F1 or anti-actin antibodies. Actin was used a protein loading control.

(Figures 6d and e). Significantly, ectopic overexpression of both TRIM16 and E2F1 into BE(2)-C cells partially blocked the inhibitory effect of TRIM16 on cell proliferation, compared with TRIM16-transfected cells alone $(P=0.002)$ (Figure 5f). These results suggested that TRIM16's effects on neuroblastoma cell growth required nuclear translocation of TRIM16 protein, binding to $\mathrm{E} 2 \mathrm{~F} 1$ protein with a consequent reduction in nuclear E2F1 levels.

To map the interaction domains of TRIM16 and E2F1, GFP-tagged TRIM16 domains and Flag-tagged E2F1 were used in co-IP experiments. The coiled-coil domain of TRIM16 is required for TRIM16 and E2F1 interaction, but not the two B-Box domains or the B30.2 domain (Figure 7a). Next, we used the cycloheximideblocking method to determine whether TRIM16 expression induces proteolysis of E2F1. These experiments were performed by transfecting $\mathrm{BE}(2)-\mathrm{C}$ cells with plasmids expressing E2F1 or TRIM16 full-length, or TRIM16 deletion mutants. As expected, E2F1 decayed at faster rates in the presence of full-length TRIM16 than in its absence (Figure 7b). The half-life of E2F1 was reduced from greater than $4 \mathrm{~h}$ to less than $2 \mathrm{~h}$. We observed that the half-life of E2F1 was also reduced in the presence of TRIM16 mutant 1 and mutant 3, but not
TRIM16 mutant 2, which suggested that the effect of TRIM16 on the degradation of E2F1 was mediated by the coiled-coil domain of TRIM16.

\section{Discussion}

Arrested tissue differentiation is a key causal feature of many human cancer types. Differentiation therapy for cancer offers the significant advantages of tissue specificity, high efficacy and low toxicity. A better understanding of the molecular mediators of the differentiation response in cancer cells will lead to an improved therapeutic index compared with general cytotoxic drugs. TRIM16 has an important role in the differentiation response of neuroblastoma cells. As a general rule, TRIM proteins appear to function as a component of large multi-protein complexes (Short et al., 2006). Our previous study had shown that TRIM16 is a nuclear transcription factor with essential effects on the retinoid anticancer signal through transcriptional regulation of retinoic acid receptor- $\beta_{2}$. In this study we show that TRIM16 demonstrates the properties of a tumour-suppressor protein. Surprisingly, 
TRIM16 bound key target proteins in both the cytoplasm and nucleus, which lead to reduced levels of cytoplasmic vimentin and nuclear E2F1, and have consequent effects on cell migration and growth, respectively.

Our data demonstrated that in neuroblasts, TRIM16 promoted neuritic differentiation. Neurogenesis involves both proliferation and differentiation. Human neuroblastoma cells show enhanced numbers of neurites and longer processes in response to aRA. The mechanism whereby retinoids regulate neurite outgrowth includes, but is not limited to, the regulation of the transcription of neurotrophin receptors (Encinas et al., 2000). More recent evidence supports a role for aRA in regulating components of other signalling pathways or candidate neurite-regulating factors. Some of these effects, such as that on neuron navigator 2, may be direct, whereas others may be secondary to other aRA-induced changes in the cell (Muley et al., 2008). In this study, our data have demonstrated that overexpression of TRIM16 led to a significant increase in the percentage of neuritebearing cells during aRA treatment, and suggested that TRIM16 is a novel candidate neurite-regulating factor and may have an important role in retinoid-induced neuronal differentiation.

TRIM proteins form large protein complexes through self-interaction, which is most likely mediated via the B box and coiled-coil domain. However, they also bind to several other proteins (Kim et al., 1996; Urano et al., 2002). In this study, we identified vimentin as a novel binding partner of TRIM16 in neuroblastoma and lung cancer cells. At the protein level, TRIM16 expression correlates inversely with vimentin expression. Vimentin is initially expressed in nearly all neuronal precursors in vivo, and is replaced by neurofilaments shortly after the immature neurons become post-mitotic, and vimentin is essential for neuritogenesis in culture (Yabe et al., 2003). In breast cancer cells, the transition from epithelial keratin to mesenchymal vimentin expression marks an important step in the malignant progression of breast cancer cells (Vora et al., 2009). Our data showed that downregulation of vimentin was required for TRIM16's effects on cell motility, indicating that loss of TRIM16 function or expression may increase the invasiveness of neuroblastoma cells. Because overexpression of TRIM16 did not change the vimentin mRNA expression level, it is probable that TRIM16 regulated vimentin at the post-translational level. In the scratch wound assay, overexpression of vimentin alone did not affect wound filling due to the presence of abundant endogenous vimentin in the cells. When both proteins are co-expressed, overexpression of TRIM16 downregulates endogenous vimentin expression, and therefore, overexpressed vimentin interferes with TRIM16's effect on cell mobility, but not with its effect on cell proliferation.

Similar to PML and hemopoietic lineage switch 5, which are members of the TRIM protein family $(\mathrm{Mu}$ et al., 1997; Lalonde et al., 2004), TRIM16 was able to reduce the growth of neuroblastoma cells, inhibit colony formation and suppress tumour development, indicating that TRIM16 is a tumour suppressor protein. Elevated levels of TRIM16 can reduce cell growth, indicating that TRIM16 might directly regulate the cell cycle machinery. As a step towards understanding the role of TRIM16 in cell cycle progression, E2F1 was identified as a novel TRIM16 binding partner and its expression is inversely correlated with TRIM16 expression in the nucleus. The E2F family of transcription factors are centrally important in mammalian cell division. E2Fs are also targets of a variety of oncogenes and tumour suppressor genes (Trimarchi et al., 2002). Therefore, it is likely that the TRIM16 and E2F1 interactions are linked to TRIM16's tumour suppression function. Furthermore, the E2F transcription factor is best known for its ability to regulate cell cycle progression by coordinating a large group of genes involved in G1-to-S phase transition. The hypophosphorylated retinoblastoma protein $(\mathrm{pRb})$ repressed $\mathrm{E} 2 \mathrm{~F}$ activity by shielding the E2F transactivation domain, leading to restriction of cell cycle progression (Weinberg, 1995). Our previous data provided evidence that TRIM16 overexpression correlated with inhibited cell growth through its effects on cyclin D1 and phospho-Rb (Raif et al., 2009). As deregulation of E2F transcriptional activity due to alterations in the $\mathrm{p} 16(\mathrm{INK} 4 \mathrm{a})-$ cyclin $\mathrm{D} 1-\mathrm{Rb}$ pathway is a hallmark of human cancer (Johnson et al., 2006), our findings suggest that TRIM16 may have a critical role in cell cycle progression, and that the effect of TRIM16 on cell proliferation may be through translocation to the nucleus, interaction with E2F1 and downregulation of nuclear E2F1 in neuroblastoma cells.

The TRIM/RBCC proteins self-associate, mainly through their coiled-coil region. Homo-interaction results in the formation of large protein complexes, which identify different discrete nuclear and/or cytoplasmic sub-cellular compartments (Reymond et al., 2001). The presence of the RING domain and its strong association with ubiquitination suggest a role for this protein family in the ubiquitin-mediated proteolysis. The RING finger domain has been found to have a critical role in mediating the transfer of ubiquitin both to heterologous substrates, as well as to the RING proteins themselves. This domain is therefore a characteristic signature of many E3 ubiquitin ligases (Joazeiro and Weissman, 2000). TRIM16 is missing the RING finger domain, but it has both B-box 1 and B-box2, which share a similar but distinct pattern of cysteine and histidine residues (Borden et al., 1993). Accumulating evidence suggests that B-boxes mediate protein-protein interactions specific to each TRIM class (Peng et al., 2000; Short et al., 2002; Mrosek et al., 2008). It is unclear how overexpressed TRIM16 reduced vimentin and E2F1 protein levels. It is possible that TRIM16 acts as an E3 ubiquitin ligase, and targets these proteins for proteolysis and promotes growth inhibition in neuroblastoma cells.

TRIM16 is expressed in most normal and cancer cells, but is mainly localized to the cytoplasmic component of cancer cells. In this study we show that TRIM16 acts as a tumour suppressor by its inhibitory effects on proteins in both the nuclear and cytoplasmic compartments. These data suggest that TRIM16 may have transactiva- 
tion-dependent effects and transactivation-independent effects. In agreement with our observations are the recent findings that $\mathrm{p} 53$ can act as a tumour suppressor in both cytoplasmic and nuclear compartments of cancer cells. In addition to being a nuclear transcription factor regulating genes involved in apoptosis and cell cycle, p53 has additional activities in the cytoplasm, where it triggers apoptosis and inhibits autophagy (Green et al., 2009).

As TRIM16 has been demonstrated to suppress tumour growth, cell migration and sensitize cancer cells to retinoids, our findings highlight TRIM16 as a potential drug development target for the treatment of cancers overexpressing vimentin and $\mathrm{E} 2 \mathrm{~F} 1$ proteins. Agents that increase the cytoplasmic and/or nuclear levels of TRIM16 might be expected to inhibit cell migration and replication, respectively, and, moreover, enhance the anticancer effects of differentiation therapies, such as retinoids.

\section{Materials and methods}

Plasmid construction and stable transfection

The TRIM16 full-length cDNA was subcloned into the pcDNA3.1(-)/myc.His vector at the EcoRI multi-cloning site (Cheung et al., 2006). The TRIM16-overexpressed BE(2)-C stable cell lines were established with transfection of pcDNA3.1(-)/TRIM16/myc-His vector or the pcDNA/mycHis empty vector. The two clones of overexpression of TRIM16 (TRIM16-4 and TRIM16-5) and two empty vector clones (EV-1 and EV-8) were selected for further studies.

The TRIM16-GFP deletion mutants were produced by OriGene (Rockville, MD, USA). The deletion mutants were synthesized by PCR of full-length TRIM16 cDNA (NM_006470.3). The final mutants were in the pCMV6-ACGFP vector. The mutant products correspond to the following amino acid (AA) sequences; AA 1-564 (full-length), AA 161-564 (M1), AA 290-564 (M2) and AA 1-372 (M3).

\section{Immunohistochemistry}

Paraffin-embedded section of neuroblastoma patients' tissue was obtained from Paediatric Anatomical Pathology, South Eastern Area Laboratory Services (SEALS). The slides were incubated with rabbit anti-TRIM16 (Bethyl Laboratories, Montgomery, TX, USA) at $1 \mu \mathrm{g} / \mathrm{ml}$ or rabbit anti-class III $\beta$-tubulin at 1 in 1000 dilution overnight at $4{ }^{\circ} \mathrm{C}$. The secondary antibody used was goat anti-rabbit immunoglobulin/biotinylated (Dako, Glostrup, Denmark) at 1 in 500 dilutions for $1 \mathrm{~h}$ at room temperature. The biotinylated antibody was then labelled with streptavidin-HRP (Dako) for $45 \mathrm{~min}$ at room temperature. The sections were developed with 3,3'-diaminobenzidine tetrahydrochloride (Dako) and counterstained with haematoxylin.

\section{Immunohistochemistry quantification}

Among the 21 neuroblastoma tumours, there were three in stage I, six in stage II, four in stage III, seven in stage IV and one in stage IVS, by the Evans staging system (Evans et al., 1971). The positive staining of TRIM16 was analysed on digitally captured images using the Aperio ScanScope XT slide scanner (Aperio Technologies, Vista, CA, USA) (Brennan et al., 2008; Rexhepaj et al., 2008). At least three of each differentiated $(\geqslant 5 \%$ of neuroblasts toward ganglion cells) and poorly differentiated regions $(\leqslant 5 \%$ of neuroblasts toward ganglion cells) with an area of minimum $10000 \mu \mathrm{m}^{2}$ were selected and analysed using colocalization algorithms with ImageScope (Aperio Technologies) (Joshi, 2000).

\section{Tumourigenicity assays}

Tumourigenicity was determined by injecting $5 \times 10^{6}$ of TRIM16 or empty vector stably tranfected $\mathrm{BE}(2)-\mathrm{C}$ cells subcutaneously into 6-8-week-old female BALB/c nude mice. Tumour volumes were measured twice a week using callipers, and the tumour mass was determined after 4 weeks post engraftment.

Transient transfection with plasmid DNA or siRNA

Cells $\left(1 \times 10^{6}\right)$ were transfected with either $15 \mu \mathrm{g}$ of TRIM16 expression vector or empty vector for $72 \mathrm{~h}$ before harvesting for transient transfection experiments. Cells were transfected with either $20 \mathrm{~nm}$ control siRNA (Dhamarcon, Lafayette, CO, USA), or siRNA specific to TRIM16 (Ambion, Foster City, CA, USA) or E2F1 (Dhamarcon) before harvesting for siRNA gene knockdown experiments.

\section{Confocal fluorescence microscopy images}

The BE(2)-C cells were fixed in 10\% formalin, and then nonspecific staining was blocked in $10 \%$ fetal calf serum/ phosphate-buffered saline. The cells were incubated in 5\% fetal calf serum/phosphate-buffered saline with goat antivimentin antibody (1:400) and custom TRIM16 rabbit antibody (1:100). The cells were then incubated with Alexa Fluor 555 anti-goat IgG (1:1000) and Alexa Fluor 488 anti-rabbit IgG (1:1000), the cells were then mounted in VectaShield with 4',6-diamidino-2-phenylindole. Images were collected with a Zeiss LSM 510 META confocal fluorescence microscope (Zeiss, Jena, Germany) using a $\times 63$ oil Plan-apochromat objective (NA, 1.4; Zeiss)

\section{Scratch wound assays and migration assay}

For scratch wound assays, BE(2)-C cells were transfected with either $15 \mu \mathrm{g}$ of TRIM16 expression vector or empty vector with Lipofectamine 2000. For the double transfection assays, $10 \mu \mathrm{g}$ of TRIM16 expression vector and $10 \mu \mathrm{g}$ of vimentin expression vector were transfected into the cells. After $48 \mathrm{~h}$ from transfection, cells were grown to $90-100 \%$ confluence and wounded using $20 \mu \mathrm{l}$ pipette tips, and the distance between the wound was measured with Image $\mathbf{J}$ software (National Institutes of Health, Bethesda, MD, USA) at various time points.

For migration assay, $\mathrm{BE}(2)-\mathrm{C}$ cells were serum starved for $2 \mathrm{~h}$ and then $4 \times 10^{4}$ cells were seed on Transwell inserts pre-coated with collagen IV on the underside of the insert. Transwell inserts were placed in companion plates containing media with $10 \%$ fetal calf serum and the cells were allowed to migrate at $37^{\circ} \mathrm{C}$. The migration index is the percentage of the migrated cells divided by the total number of cells in the wells.

\section{Immunoprecipitation assays and western blots}

Lysates were immunoprecipitated with anti-TRIM16 (Bethyl Laboratories) or anti-myc tag antibody (Cell Signalling Technology, Danvers, MA, USA). Anti-vimentin antibody, anti-E2F1 antibody and anti-Myc tag antibody were used in immunoblots. Rabbit polyclonal actin antibody (Sigma, St Louis, MO, USA), and Histone H3 antibody and antiGAPDH antibody were used to normalize for differences in whole cell lysates, nuclear or cytoplasmic protein loading, respectively. 


\section{Conflict of Interest}

The authors declare no conflict of interest.

\section{Acknowledgements}

This research was supported by programme grants from the National Health and Medical Research Council, Australia.

\section{References}

Beer HD, Munding C, Dubois N, Mamie C, Hohl D, Werner S. (2002). The estrogen-responsive B box protein: a novel regulator of keratinocyte differentiation. J Biol Chem. 277: 20740-20749.

Borden KL, Martin SR, O'Reilly NJ, Lally JM, Reddy BA, Etkin LD et al. (1993). Characterisation of a novel cysteine/histidine-rich metal binding domain from Xenopus nuclear factor XNF7. FEBS Letters 335: 255-260.

Brennan DJ, Rexhepaj E, O'Brien SL, McSherry E, O'Connor DP, Fagan A et al. (2008). Altered cytoplasmic-to-nuclear ratio of survivin is a prognostic indicator in breast cancer. Clin Cancer Res 14: 2681-2689.

Cao T, Duprez E, Borden KL, Freemont PS, Etkin LD. (1998). Ret finger protein is a normal component of PML nuclear bodies and interacts directly with PML. J Cell Sci 111: 1319-1329.

Chae JJ, Komarow HD, Cheng J, Wood G, Raben N, Liu PP et al. (2003). Targeted disruption of pyrin, the FMF protein, causes heightened sensitivity to endotoxin and a defect in macrophage apoptosis. Mol Cell 11: 591-604.

Cheung BB, Bell J, Raif A, Bohlken A, Yan J, Roediger B et al. (2006). The estrogen-responsive $\mathrm{B}$ box protein is a novel regulator of the retinoid signal. J Biol Chem 281: 18246-18256. (e-pub ahead of print 24 April 2006).

Encinas M, Iglesias M, Liu Y, Wang H, Muhaisen A, Cena V et al. (2000). Sequential treatment of SH-SY5Y cells with retinoic acid and brain-derived neurotrophic factor gives rise to fully differentiated, neurotrophic factor-dependent, human neuron-like cells. $J$ Neurochem 75: 991-1003.

Evans AE, D'Angio GJ, Randolph J. (1971). A proposed staging for children with neuroblastoma. Children's cancer study group A. Cancer 27: 374-378.

Green DR, Kroemer G, Green DR, Kroemer G. (2009). Cytoplasmic functions of the tumour suppressor p53. Nature 458: 1127-1130.

Joazeiro CA, Weissman AM. (2000). RING finger proteins: mediators of ubiquitin ligase activity. Cell 102: 549-552.

Johnson DG, Degregori J. (2006). Putting the oncogenic and tumor suppressive activities of E2F into context. Curr Mol Med 6: 731-738.

Joshi VV. (2000). Peripheral neuroblastic tumors: pathologic classification based on recommendations of international neuroblastoma pathology committee (modification of shimada classification). Pediatr Dev Pathol 3: 184-199.

Katsetos CD, Herman MM, Frankfurter A, Uffer S, Perentes E, Rubinstein LJ. (1991). Neuron-associated class III beta-tubulin isotype, microtubule-associated protein 2 , and synaptophysin in human retinoblastomas in situ. Further immunohistochemical observations on the Flexner-Wintersteiner rosettes. Lab Invest 64: 45-54.

Kim SS, Chen YM, O'Leary E, Witzgall R, Vidal M, Bonventre JV. (1996). A novel member of the RING finger family, KRIP-1, associates with the KRAB-A transcriptional repressor domain of zinc finger proteins. Proc Natl Acad Sci USA 93: 15299-15304.

Lalonde JP, Lim R, Ingley E, Tilbrook PA, Thompson MJ, McCulloch $\mathrm{R}$ et al. (2004). HLS5, a novel RBCC (ring finger, B box, coiled-coil) family member isolated from a hemopoietic lineage switch, is a candidate tumor suppressor. J Biol Chem 279: 8181-8189.

Le Douarin B, Zechel C, Garnier JM, Lutz Y, Tora L, Pierrat P et al. (1995). The N-terminal part of TIF1, a putative mediator of the ligand-dependent activation function (AF-2) of nuclear receptors,
This research project was also supported by an Early Career Development Fellowship from the Cancer Institute NSW. The Children's Cancer Institute Australia for Medical Research is affiliated with the University of NSW and Sydney Children's Hospital. We thank Ms Tala Kaplinovsky of the Westfield Research Laboratory at Sydney Children's Hospital for help with using confocal microscopy. We thank $\mathrm{Mr}$ Andrew Gayagay of the Paediatric Anatomical Pathology, SEALS for help with preparation of patient samples. is fused to B-raf in the oncogenic protein T18. EMBO $J$ 14: 2020-2033.

Liu HL, Golder-Novoselsky E, Seto MH, Webster L, McClary J, Zajchowski DA. (1998). The novel estrogen-responsive B-box protein (EBBP) gene is tamoxifen-regulated in cells expressing an estrogen receptor DNA-binding domain mutant. Mol Endocrinol 12: $1733-1748$

Matthay KK, Reynolds CP, Seeger RC, Shimada H, Adkins ES, HaasKogan D et al. (2009). Long-term results for children with high-risk neuroblastoma treated on a randomized trial of myeloablative therapy followed by 13-cis-retinoic acid: a children's oncology group study. J Clinl Oncol 27: 1007-1013.

Matthay KK, Villablanca JG, Seeger RC, Stram DO, Harris RE, Ramsay NK et al. (1999). Treatment of high-risk neuroblastoma with intensive chemotherapy, radiotherapy, autologous bone marrow transplantation, and 13-cis-retinoic acid. Children's Cancer Group. N Engl J Med 341: 1165-1173.

McInroy L, Maatta A. (2007). Down-regulation of vimentin expression inhibits carcinoma cell migration and adhesion. Biochem Biophys Res Commun 360: 109-114.

Meroni G, Diez-Roux G. (2005). TRIM/RBCC, a novel class of 'single protein RING finger' E3 ubiquitin ligases. Bioessays 27: $1147-1157$.

Mrosek M, Meier S, Ucurum-Fotiadis Z, von Castelmur E, Hedbom E, Lustig A et al. (2008). Structural analysis of B-Box 2 from MuRF1: identification of a novel self-association pattern in a RING-like fold. Biochemistry 47: 10722-10730

Mu ZM, Le XF, Vallian S, Glassman AB, Chang KS. (1997). Stable overexpression of PML alters regulation of cell cycle progression in HeLa cells. Carcinogenesis 18: 2063-2069.

Muley PD, McNeill EM, Marzinke MA, Knobel KM, Barr MM, Clagett-Dame M. (2008). The atRA-responsive gene neuron navigator 2 functions in neurite outgrowth and axonal elongation. Dev Neurobiol 68: 1441-1453.

Peng H, Begg GE, Harper SL, Friedman JR, Speicher DW, Rauscher III FJ. (2000). Biochemical analysis of the Kruppel-associated box (KRAB) transcriptional repression domain. J Biol Chem 275 18000-18010.

Raif A, Marshall GM, Bell JL, Koach J, Tan O, D'Andreti C et al (2009). The estrogen-responsive B box protein (EBBP) restores retinoid sensitivity in retinoid-resistant cancer cells via effects on histone acetylation. Cancer Lett 277: 82-90.

Rexhepaj E, Brennan DJ, Holloway P, Kay EW, McCann AH, Landberg $G$ et al. (2008). Novel image analysis approach for quantifying expression of nuclear proteins assessed by immunohistochemistry: application to measurement of oestrogen and progesterone receptor levels in breast cancer. Breast Cancer Res 10: R89.

Reymond A, Meroni G, Fantozzi A, Merla G, Cairo S, Luzi L et al. (2001). The tripartite motif family identifies cell compartments. EMBO J 20: $2140-2151$.

Short KM, Cox TC. (2006). Subclassification of the RBCC/TRIM superfamily reveals a novel motif necessary for microtubule binding. JBiol Chem 281: 8970-8980.

Short KM, Hopwood B, Yi Z, Cox TC et al. (2002). MID1 and MID2 homo- and heterodimerise to tether the rapamycin-sensitive PP2A regulatory subunit, alpha 4 , to microtubules: implications for the 
clinical variability of X-linked Opitz GBBB syndrome and other developmental disorders. BMC Cell Biol 3: 1.

Thiele CJ, Reynolds CP, Israel MA. (1985). Decreased expression of $\mathrm{N}$-myc precedes retinoic acid-induced morphological differentiation of human neuroblastoma. Nature 313: 404-406.

Trimarchi JM, Lees JA. (2002). Sibling rivalry in the E2F family. Nat Rev Mol Cell Biol 3: 11-20.

Trockenbacher A, Suckow V, Foerster J, Winter J, Krauss S, Ropers HH et al. (2001). MID1, mutated in Opitz syndrome, encodes an ubiquitin ligase that targets phosphatase $2 \mathrm{~A}$ for degradation. [erratum appears in Nat Genet 2002 Jan;30(1):123]. Nat Genet 29: 287-294.

Urano T, Saito T, Tsukui T, Fujita M, Hosoi T, Muramatsu M et al. (2002). Efp targets 14-3-3 sigma for proteolysis and promotes breast tumour growth. Nature 417: 871-875.

Vichi A, Payne DM, Pacheco-Rodriguez G, Moss J, Vaughan M, Vichi A et al. (2005). E3 ubiquitin ligase activity of the trifunctional ARD1 (ADP-ribosylation factor domain protein 1). ProcNatl Acad Sci USA 102: 1945-1950.

Vora HH, Patel NA, Rajvik KN, Mehta SV, Brahmbhatt BV, Shah MJ et al. (2009). Cytokeratin and vimentin expression in breast cancer. Int J Biol Markers 24: 38-46.

Weinberg RA. (1995). The retinoblastoma protein and cell cycle control. Cell 81: 323-330.

Weiss WA, Aldape K, Mohapatra G, Feuerstein BG, Bishop JM. (1997). Targeted expression of MYCN causes neuroblastoma in transgenic mice. EMBO J 16: 2985-2995.

Yabe JT, Chan WK, Wang FS, Pimenta A, Ortiz DD, Shea TB et al. (2003). Regulation of the transition from vimentin to neurofilaments during neuronal differentiation. Cell Motil Cytoskeleton 56: 193-205.

(c)

SOMERIEHTS RES

This work is licensed under the Creative Commons Attribution-NonCommercial-No Derivative Works 3.0 Unported License. To view a copy of this license, visit http://creativecommons.org/licenses/by-nc-nd/3.0/ 